\title{
BMJ Global Health Comparative analysis of gender differences in outcomes after trauma in India and the USA: case for standardised coding of injury mechanisms in trauma registries
}

\author{
Mohini Dasari, ${ }^{1}$ Siddarth D David, ${ }^{2}$ Elizabeth Miller, ${ }^{3}$ Juan Carlos Puyana, ${ }^{1}$ \\ Nobhojit Roy ${ }^{4}$
}

To cite: Dasari M, David SD, Miller $\mathrm{E}$, et al. Comparative analysis of gender differences in outcomes after trauma in India and the USA: case for standardised coding of injury mechanisms in trauma registries. BMJ Glob Health 2017;2:e000322. doi:10.1136/ bmjgh-2017-000322

Received 22 February 2017 Revised 30 May 2017 Accepted 6 June 2017

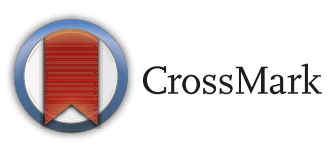

${ }^{1}$ Department of Surgery, University of Pittsburgh School of Medicine, Pittsburgh, Pennsylvania, USA

${ }^{2}$ School of Habitat Studies, Tata Institute of Social Sciences, Deonar, India

${ }^{3}$ Division of Young Adult and Adolescent Medicine, University of Pittsburgh School of Medicine, Pittsburgh,

Pennsylvania, USA

${ }^{4}$ Department of Public Health Sciences, School of Habitat Studies, Tata Institute of Social Sciences Karolinska Institutet, Stockholm, Sweden

Correspondence to Mohini Dasari; mod13@pitt.edu

\section{ABSTRACT}

Introduction While females generally have better outcomes than males after traumatic injury, higher mortality has been shown to occur in females after intentional trauma in lower-income countries. However, gender differences in trauma outcomes in different countries have not been previously compared. We conducted a two-country comparative analysis to characterise gender differences in mortality for different mechanisms of injury.

Methods Two urban trauma databases were analysed from India and the USA for fall, motor vehicle collision (MVC) and assault patients between 2013 and 2015. Coarsened exact matching was used to match the two groups based on gender, age, injury severity score, Glasgow Coma Score and type of injury (blunt vs penetrating). The primary outcome of mortality was studied by using logistic regression to calculate the odds of death in the four country/gender subgroups.

Results A total of 10089 and 14144 patients were included from the Indian and US databases, respectively. After matching on covariates, 7505 and 9448 patients were included in the logistic regression. Indian males had the highest odds of death compared with US males, US females and Indian females for falls, MVC and assaults. Indian females had over 7 times the odds of dying after falls, 5 times the odds of dying for MVC and 40 times the odds of dying after assaults when compared with US females. Conclusion The high odds of death for Indian females compared with US females suggests that there are other injury and systemic factors that contribute to this discrepancy in mortality odds. This same mortality pattern and implication is seen for Indian males compared with all subgroups. Standardised coding of injury mechanism in trauma registries, in addition to intentionality of injury, can help further characterise discrepancies in outcomes by gender and country, to guide targeted injury prevention and care.

\section{INTRODUCTION}

Over $90 \%$ of traumatic injuries occur in low-middle income countries, yet most of the data regarding the presentation and outcomes of patients in these countries are not captured in registries. ${ }^{1-3}$ Trauma registries are a critical component of monitoring outcomes and directing quality improvement, especially in low-resource settings. ${ }^{4}$ Several comparative studies have been conducted to analyse factors leading to discrepancies in trauma outcomes between high and lower-income countries. $^{5-7}$ Injury mechanism, differences in clinical interventions and the presence or absence of regional trauma systems have been cited as some of the elements contributing to observed differences in outcomes. ${ }^{5-7}$ Comparing outcomes in different countries can help guide injury prevention and trauma care improvement initiatives. ${ }^{6}$ However, there have been no prior studies examining gender differences in trauma outcomes between countries. Thus, knowledge of how to approach gender-based differences in trauma outcomes on a global scale is lacking.

Gender differences after trauma have been previously studied, mostly in the USA. Females in the USA have been shown to have fewer complications and lower mortality after trauma than similarly injured males in a nationwide sample. ${ }^{8}$ A state-based study confirmed that female gender did not adversely affect mortality after adjusting for age and injury severity. ${ }^{9}$ A hormonal basis has even been implicated in the observed better outcomes after trauma-associated sepsis in females compared with males in the USA. ${ }^{10}$ However, such detailed analyses of gender differences after trauma have been limited to higher-income countries such as the USA.

In lower/middle income countries (LMICs), several retrospective studies have revealed significantly higher mortality among female 


\section{Summary Box}

\section{What is already known about this topic?}

- Ninety per cent of deaths due to trauma worldwide occurs in lower/ middle income countries (LMICs).

- Higher mortality from trauma in LMICs attributed to differences in injury mechanisms, and the presence or absence of prehospital and trauma systems.

- Males usually have worse morbidity and mortality following trauma than females in the USA.

- Females often have worse mortality than males in lower-income countries after intentional trauma.

\section{What are the new findings?}

- Females in India who were assaulted had nearly 40 times the odds of mortality compared with similarly injured female assault patients in the USA, accounting for the largest difference in odds of mortality between either gender in either country.

- Males and females in the higher-income country (USA) usually had five to seven times lower odds of mortality than their similarly injured counterparts in a lower-income country (India) for falls and motor vehicle collisions.

- Indian males had the highest overall odds of mortality for all mechanisms of injury among all genders and both countries.

\section{Recommendations for policy}

- Our findings highlight an important, previously unpublished discrepancy in gender outcomes after trauma, especially for female patients who presented for trauma care after sustaining assault.

- Trauma clinical care providers, including developing/existing prehospital care systems, should be aware of the potential social and/or societal reasons for this mortality discrepancy.

- Our findings support the need for trauma registries to develop standardised coding mechanisms for mechanism and intentionality of injury in order to elucidate additional reasons for mortality discrepancies that may not be related to clinical care or injury severity.

patients with trauma compared with males, most notably among patients with burn. ${ }^{11-13}$ However, these studies have not adjusted for demographics and injury severity in making comparisons between males and females. Additionally, there have been no studies examining mortality across gender based on mechanism of injury in different countries. Studying gender differences after trauma in different countries is an important step towards improved injury prevention, given that gender-based violence is prevalent in both high-income and lower-income countries. ${ }^{14}$ Given this, our aim was to conduct a two-country comparative analysis to characterise gender differences in mortality for different mechanisms of injury between a high-income and lower-income country.

\section{METHODS}

\section{Description of databases}

The two trauma databases were analysed from India and the USA. The Indian database was developed through a research consortium initiative, 'Towards Improving Trauma Care Outcomes' (India), and contains exclusively data of patients with trauma from four hospitals in the three largest megacities (population $>10$ million inhabitants) in India: Kolkata (Seth Sukhlal Karnani Memorial Hospital), Mumbai (King Edwards Memorial Hospital and Lokmanya Tilak Municipal General Hospital) and Delhi (the Apex Trauma Centre of the All-India Institute of Medical Sciences). The first two are large public hospitals that provide care, including trauma, to the lower socioeconomic strata. The Apex Trauma Centre is a public-funded standalone trauma hospital. The database contains data from 2013 to 2015. There are other public hospitals in each of the cities which are not part of the study, and thus the patients are a proportion of all the patients with trauma in the three cities. However, as the three included are the major public hospitals in the three cities, they share a large burden of the patients with trauma.

The US database is from one of three level 1 trauma centres in Pittsburgh, Pennsylvania, a medium-size city with over 305000 inhabitants. The database contains all patients with trauma admitted to this centre, with the exception of patients with burn, who are seen at a different trauma centre in the city, and any patient who was dead at the scene prior to transport to the hospital. The trauma centre is one of two major centres in Pittsburgh, and receives the majority of the trauma in the city. Data were extracted from 2013 to 2015 to match the study period of the Indian database. The US database is a single-centre database versus the Indian database which is multicentre.

\section{Data}

Variables common to both databases extracted for analysis were age, gender, Injury Severity Score (ISS), Glasgow Coma Scale (GCS), Revised Trauma Score (RTS), type of injury (blunt vs penetrating) and in-hospital mortality. In the case of intubated patients, the verbal part of the GCS was recorded as for one in the Indian cohort. The same was done in the US database. The top three mechanisms of injury were analysed: falls, motor vehicle collisions (MVC) and assaults. In the Indian registry, this had been precoded as 'Mechanism of Injury' with the following options: fall, railway collision, road traffic collision, assault, burn, other. In the US database, there was no specific categorical variable for mechanism of injury. Thus, we generated a binary variable to represent those three types of injury by including all patients who had an ICD-9 E code (International Classification of Diseases, External Causes of Injury) whose Manner/Intent was either a fall, MVC or assault. The former two included both unintentional and intentional mechanisms under the same variable.

\section{Statistical analysis}

Statistical analyses were performed using Stata 14 (Stata 2015. Stata Statistical software: Release 14). Variables were summarised by country and gender using descriptive statistics. Variables were then compared using $\mathrm{X}^{2}$ for categorical variables and Kruskal-Wallis for continuous 
variables. We examined all variables between the two countries, with subanalyses according to gender. All analyses were carried out with a $95 \%$ confidence level ( $p$ value $<0.05$ considered significant).

The coarsened exact matching (CEM) method was employed in order to achieve a rough match between the two databases prior to comparative analysis. ${ }^{15}$ Given that the nature of the populations being analysed were so different at baseline, in terms of demographic variables and injury severity, this method was chosen instead of a traditional one-to-one matching method. CEM matches two datasets based on user-determined ranges of values for each variable, which are called strata. ${ }^{15}$ CEM matches observations that are within the same strata for every variable that is included in the match. Thus, CEM ensures that matched observations, in this case, patients, have values within a similar range for each covariate of interest. The quality of the match achieved after CEM can be evaluated using the L1 parameter, which is a marker of imbalance between two groups in terms of their covariates. Thus, a decrease in L1 indicates improvement in imbalance between the group groups after matching..$^{15}$ Of note, CEM only matches subjects that do not have missing values for any of the specific variables; thus, if subjects are missing data points for any of the variables, they are excluded from the match. This precluded the need for a method to handle missing data values, as it is inherently part of the CEM algorithm.

The datasets were matched on the following variables: gender, age, ISS, GCS and type of injury (blunt vs penetrating). We employed logistic regression with the primary outcome of mortality, adjusted for the variables on which the databases were already matched. The results of the logistic regression model were used to calculate the odds of death in the four country/gender subgroups.

\section{RESULTS}

Between 2013 and 2015, the Indian and US databases contained 11670 and 14155 patients, respectively. After exclusion of railway and burn injuries, there were 10089 Indian and 14144 US patients included. The coarsened exacting matching procedure resulted in a matched cohort of 7505 Indian and 9448 US patients. The multivariate $\mathrm{L} 1$ distance was 0.891 before matching and 0.601 after matching, indicating improvement in imbalance between the two groups after matching. Both groups still remained significantly different across most variables before and after CEM (table 1).

On examining the differences between males and females in each country across key demographic and outcome variables, gender differences were found in the prevalence of blunt versus penetrating injury, the prevalence of surgical intervention after trauma, days of ventilation and trauma mortality (table 2). Notably, males incurred significantly more trauma than females in both countries $\left(X^{2}\right.$ test $p$ value $<0.05$, table 2$)$. Indian males had significantly higher rates of surgical intervention than Indian females, while US females had significantly higher rates of surgical intervention than US males (table 2). US males had significantly longer ventilation time periods than US females, and had significantly higher mortality rates than US females as well $(3.3 \%$ vs $2.5 \%$, table 2). While overall mortality among Indian

Table 1 Baseline characteristics by country in entire and matched cohorts

\begin{tabular}{|c|c|c|c|c|c|c|}
\hline & \multicolumn{3}{|c|}{ Entire cohort } & \multicolumn{3}{|c|}{ Matched cohort } \\
\hline & India & USA & p Value & India & USA & p Value \\
\hline$n$ & 10089 & 14144 & & 7505 & 9448 & \\
\hline Sex, female* & $2058(20.4)$ & 5637 (39.9) & $<0.001$ & $1356(18.1)$ & 3141 (33.2) & $<0.001$ \\
\hline Sex, male* & 8030 (79.6) & $8507(60.1)$ & $<0.001$ & $6149(81.9)$ & 6307 (66.8) & $<0.001$ \\
\hline Age* $^{*}$ & $32.9 \pm 19.2$ & $53.1 \pm 22.6$ & $<0.0001$ & $32.9 \pm 20$ & $52.8 \pm 24$ & $<0.0001$ \\
\hline ISS* & $11 \pm 7$ & $8 \pm 7$ & $<0.0001$ & $10 \pm 6$ & $8 \pm 6$ & $<0.001$ \\
\hline $\mathrm{RTS}^{*}$ & $4.08 \pm 1.6$ & $7.64 \pm 0.9$ & $<0.0001$ & $4.41 \pm 1.4$ & $7.66 \pm 0.8$ & $<0.0001$ \\
\hline GCS* & $12 \pm 3$ & $14 \pm 1$ & $<0.0001$ & $13 \pm 1$ & $14 \pm 1$ & $<0.001$ \\
\hline Blunt injury* & $9622(95.5)$ & 12744 (94.9) & 0.029 & 7203 (96.1) & 9125 (96.8) & 0.014 \\
\hline Penetrating injury* & $456(4.5)$ & $691(5.1)$ & 0.029 & $296(3.9)$ & $306(3.2)$ & 0.014 \\
\hline Surgery* & 2867 (28.5) & 710 (5.2) & $<0.001$ & $2,072(27.7)$ & 436 (4.6) & $<0.001$ \\
\hline Ventilation days* & $1.2 \pm 5.9$ & $12 \pm 2.5$ & $<0.0001$ & $1.0 \pm 6.2$ & $11 \pm 2.4$ & $<0.0001$ \\
\hline ICU days* & $1.5 \pm 6.0$ & $1.1 \pm 3.5$ & 0.3324 & $1.3 \pm 6.3$ & $1.1 \pm 3.4$ & 0.0012 \\
\hline Hospital LOS* & $7.2 \pm 12.6$ & $4.7 \pm 6.4$ & $<0.0001$ & $7.2 \pm 12.0$ & $4.6 \pm 6.2$ & $<0.0001$ \\
\hline In-hospital death* & 1928 (22.9) & $422(3.0)$ & $<0.001$ & $988(15.8)$ & $265(2.8)$ & $<0.001$ \\
\hline
\end{tabular}

Numbers reported as mean \pm SD or count (percentage).

Bolded variables were used for matching.

${ }^{*} \chi^{2}$ test for association between variable and country and Wilcoxon rank-sum test for difference in means between countries.

GCS, Glasgow Coma Score; ICU, Intensive Care Unit; ISS, Injury Severity Score; LOS, length of stay; RTS, Revised Trauma Score. 
Table 2 Baseline characteristics by sex and country in matched cohorts

\begin{tabular}{|c|c|c|c|c|c|c|}
\hline & \multicolumn{2}{|l|}{ India } & \multicolumn{3}{|c|}{ USA } & \multirow[b]{2}{*}{ p Value } \\
\hline & Females & Males & p Value & Females & Males & \\
\hline$n^{*}$ & $1356(18.1)$ & 6149 (81.9) & $<0.05$ & 3141 (33.2) & 6307 (66.8) & $<0.05$ \\
\hline $\mathrm{Age}^{*}$ & $31.2 \pm 22$ & $32.4 \pm 18$ & 0.64 & $59.6 \pm 23$ & $48.8 \pm 21$ & 0.49 \\
\hline ISS* & $11 \pm 6$ & $12 \pm 8$ & 0.19 & $8 \pm 7$ & $8 \pm 7$ & 0.15 \\
\hline $\mathrm{GCS}^{*}$ & $12 \pm 3$ & $12 \pm 3$ & 0.12 & $14 \pm 1$ & $14 \pm 1$ & 0.02 \\
\hline Blunt injury* & 1349 (95.5) & 5829 (94.8) & $<0.05$ & 1329 (98.1) & 5842 (92.6) & $<0.05$ \\
\hline Penetrating injury* & $7(4.5)$ & $320(5.2)$ & $<0.05$ & $27(1.9)$ & $465(7.4)$ & $<0.05$ \\
\hline Surgery* & $318(23.4)$ & $1892(30.8)$ & $<0.05$ & $301(9.6)$ & $385(4.7)$ & $<0.05$ \\
\hline Ventilation days* & $0.1 \pm 0.8$ & $1.5 \pm 7.6$ & 0.10 & $0.3 \pm 2$ & $0.6 \pm 2.8$ & 0.05 \\
\hline ICU days* & $0.2 \pm 8.1$ & $1.7 \pm 4.7$ & 0.23 & $0.9 \pm 2.7$ & $1.3 \pm 4.0$ & 0.07 \\
\hline Hospital LOS* & $2.9 \pm 6.5$ & $9.0 \pm 14.8$ & 0.26 & $4.5 \pm 5.1$ & $4.8 \pm 7.1$ & 0.13 \\
\hline In-hospital death* & $382(28.2)$ & 1610 (26.2) & 0.13 & $78(2.5)$ & 206 (3.3) & 0.04 \\
\hline
\end{tabular}

Numbers reported as mean \pm SD or count (percentage).

${ }^{*} \chi^{2}$ test for association between variable and gender and Wilcoxon rank-sum test for difference in means between genders within each country. $p$ Value deemed significant at alpha level<0.05.

GCS, Glasgow Coma Score; ICU, Intensive Care Unit; ISS, Injury Severity Score; LOS, length of stay; RTS, Revised Trauma Score.

females was grossly higher than among Indian males ( $28.2 \%$ vs $26.2 \%$, respectively), this difference was not statistically significant.

Within the matched cohort, odds of mortality among the four subgroups (Indian males, Indian females, US males, US females) was highest for Indian males across all three leading mechanisms of injury (table 3). Among US patients, males had roughly three times the odds of death after falls compared with females $(p<0.05)$. However, the differences in odds for mortality were not significant for MVC and assault injuries. Among Indian patients, females had lower adjusted odds of mortality compared with males across all three injury types.

\begin{tabular}{|c|c|c|c|c|}
\hline & & $\mathbf{n}$ & OR & $95 \% \mathrm{Cl}$ \\
\hline \multirow[t]{4}{*}{ Fall } & US male & 3351 & & \\
\hline & US female & 3369 & 0.36 & (0.33 to 0.39 ) \\
\hline & Indian male & 2877 & 3.68 & (3.21 to 4.22 ) \\
\hline & Indian female & 996 & 2.56 & (1.98 to 3.32 ) \\
\hline \multirow[t]{4}{*}{ MVC } & US male & 2100 & & \\
\hline & US female & 1361 & 1.05 & (0.88 to 1.25$)$ \\
\hline & Indian male & 3978 & 6.97 & (5.88 to 8.26 ) \\
\hline & Indian female & 869 & 5.23 & (3.88 to 7.05 ) \\
\hline \multirow[t]{4}{*}{ Assault } & US male & 1031 & & \\
\hline & US female & 231 & 0.13 & (0.01 to 1.10$)$ \\
\hline & Indian male & 745 & 6.06 & (3.09 to 11.85 ) \\
\hline & Indian female & 96 & 5.26 & (1.17 to 23.71 ) \\
\hline
\end{tabular}

${ }^{*}$ Reference category for OR is US male.

MVC, motor vehicle collision.
Between gender groups in both countries, Indian males and females had three to seven higher odds of mortality compared with their US counterparts for almost all mechanisms of injury (table 3). Within the assaults category, Indian females had over five times the odds of mortality compared with US males, whereas US females had only 0.13 times the odds of death compared with US males. This difference in odds of death for females in India and the US after assaults was the largest difference in odds among all subgroups and injury mechanisms.

Further characterisation of the assault injuries based on type of injury (blunt vs penetrating mechanism) revealed that Indian males with blunt assault injuries had higher mortality while Indian females with penetrating assault injuries had higher mortality (table 4). No significant difference was observed in mortality between US males and females. Indian females with penetrating assault injury had the highest mortality (13\%) despite having the lowest injury severity for penetrating injuries when compared with Indian males, as well as US males and females.

Table 4 Injury severity and mortality by gender, country and type of assault

\begin{tabular}{llllll} 
& Blunt & & & \multicolumn{2}{c}{ Penetrating } \\
\cline { 1 - 2 } \cline { 5 - 6 } Variable & ISS $^{*}$ & Died† (\%) & & ISS & Died†(\%) \\
\hline Indian males & $9.5 \pm 6$ & 10 & & $7.0 \pm 6$ & 6 \\
Indian females & $8.7 \pm 6$ & 7 & & $6.5 \pm 4$ & 13 \\
US males & $5.8 \pm 5$ & 0.2 & & $8.3 \pm 10$ & 6 \\
US females & $5.0 \pm 5$ & 0.6 & & $6.9 \pm 9$ & 5 \\
\hline
\end{tabular}

${ }^{*}$ Reported as mean $\pm \mathrm{SD}$.

†Reported as percentage died from total in that group. ISS, Injury Severity Score. 


\section{DISCUSSION}

This two-country comparative analysis of patients with trauma in India and the USA is the first study to examine differences in trauma outcomes based on gender between a high-income and lower-income country. Our findings confirm previous literature citing increased mortality after trauma in lower-income countries. ${ }^{5}$ Additionally, our matched analysis revealed significant differences in outcomes based on gender, with higher adjusted odds of mortality in males compared with females in both countries. These odds differences were significant in patients sustaining falls in the USA, and across all three mechanisms of injury in India.

Prior literature has shown that males tend to have higher adjusted morbidity and mortality after trauma compared with females. ${ }^{8}$ Whether this is due to differences in recovery after injury or differences in trauma mechanisms has not been clarified in the literature, although molecular differences in response to injury have been implicated. ${ }^{10}$ Our data suggest that these gender mortality differences persist in both higher-income and lower-income country settings. Indian males have been shown to be involved in the majority of fatal MVCs in India. ${ }^{16}$ The high odds of death for Indian males across all groups from our data implies that injury mechanisms may be significantly worse for males in a lower-income country than females in the same country and males in a higher-income country. The data, combined with established evidence about fatality from MVCs in LMICs, suggest that males may be involved in more high-speed collisions, resulting in more fatal injuries and higher mortality. Additionally, while the Indian cohort was on average about two decades younger than the US cohort, they had higher mortality; however, this is consistent with the fact that the median age of the Indian population is 27 years of age, while that of the USA is 38 years of age. ${ }^{17}$

Interestingly, after examining differences within genders between two countries, our data reveal new discrepancies. While both males and females in India had higher odds of mortality than their US counterparts, Indian female assault patients had a 40 times higher odds of death than US females (table 4). This odds difference was unparalleled among any of the other subgroups and mechanisms of injury. This finding raises several questions, many of which cannot be answered given the current limitations in how trauma registries document intentionality of injury. For instance, is this difference in mortality attributable to potential delay between injury time and medical attention, along with limited prehospital care services and access to affordable surgical care? The potential demographic and social variables contributing to this discrepancy are not currently quantified in registries in both high-income and low-income countries. In terms of social variables, there is data showing that domestic violence contributes to a significant percentage of female trauma patients presenting in high-income and low-income countries. ${ }^{18-20}$ Multiple strong sources of evidence suggest that only one in four women in India seek care services related to experiencing intimate partner violence. ${ }^{21}{ }^{22}$ While present data cannot be conclusive, time to care may indeed be prolonged in cases of intentional injury and gender-based violence.

Our study suggests that, even after adjustment for demographic and injury severity variables in a matched sample, significant differences persist between males and females in a high-income and lower-income country. The multitude of prehospital and clinical care variables that are not captured in current registries may explain these differences. For example, registries that include comprehensive complication and infection data could be used to assess which clinical factors may be leading to discrepancies in observed in-hospital mortality between genders and countries. This concepts can be encompassed under the broader concept of trauma system development that is the goal of many trauma quality improvement initiatives in lower-resource countries. ${ }^{23}$ The institution of trauma care protocols in lower-resource countries has been shown to improve clinical outcomes. ${ }^{2425}$ However, one of the largest barriers to trauma research and quality improvement in such settings is the absence of established prehospital care systems. ${ }^{26}$ This makes data collection of prehospital variables, such as delay between time of injury to hospital attention, difficult.

With recent discussion in the global trauma care community about the utility of an international trauma registry, ${ }^{27}$ we suggest that an international coding system for prehospital delay and intentional injury in trauma information systems also take place concurrently. A systematic method for screening and recording of time between injury to arrival and intentional injury is sorely needed in trauma registries, in both developed countries and LMICs. The current method of documentation, if it occurs at all, is most likely incomplete, given our inability to directly calculate intentional injury estimates from trauma registry samples. However, the collection of such data could be powerful and facilitate deeper analysis into some of the trends uncovered by our analysis, such as discrepancies between adjusted mortality for males in India compared with the USA, as well as the 40 times higher odds of mortality for females sustaining assault injuries in India compared with the USA. Ultimately, availability of prehospital services and infrastructure, and subsequently data collecting capacity, in LMICs determines whether variables such as prehospital transport request time, depart time and hospital arrival time can be collected; in India, at present, the limited presence of prehospital precludes widespread capturing of these data points.

With regard to differences in intervention between the two countries, we saw significantly higher rates of surgical management of patients with trauma in India compared to the USA (table 1). Since that the databases are not granular to this degree, we are unable to explain exactly why this is the case. In India, many MVCs and assault cases, because of the medico-legal nature of 
case, are transferred to public hospitals. Additionally, many patients are transferred to public hospitals when they cannot afford surgical treatment in other private health facilities. Since the Indian cohort is based on public trauma centres, there could be a larger number of surgeries occurring given that these centres provide more tertiary level care to those who cannot afford private care or are referred up to such larger-volume hospitals for cases requiring operative management. Given the limited use of resources such as interventional radiology in such public hospitals in India, all these cases represent surgical procedures occurring in the operating room versus bedside procedures. The same is the case in the US database. Additionally, we saw a discrepancy between ISS and GCS severity in India and the USA, with mild ISS values in India despite significantly higher mortality. Indian public hospitals have limited resources and radiological interventions such as CT, ultrasound and X-rays are not done routinely for patients with trauma unless in cases of severe injury. Hence, many internal injuries maybe missed and not recorded in the coding of ISS and GCS. Additionally, as autopsy reports are usually not used in the Indian cohort, any data internal injury findings would not be available. That is why even patients with higher ISS or higher GCS may have high mortality. Differences in specific resource utilisation for patients in different countries is a subject of much interest and relevance to comparative trauma research worldwide.

This exploratory study is not without several limitations. Given that the two databases were not standardised in the same way with regard to mechanism of injury, we had to adapt the US database to fit the mechanism of injuries coded in the Indian database, through ICD-9 subgroup coding. It is possible that there were patients coded within the Indian database as 'falls' or 'MVCs' who were intentionally injured; however, this would not have been captured given that 'assaults' was its own category. This supports the need for standardised intentionality of injury coding in trauma registries, separate from the mechanism of injury variable. Additionally, the matching achieved from our CEM method was an imperfect match. This is largely attributable to the skewed distributions of nearly all the variables, such as age, injury severity and length of stay between the two databases. Finally, we are not able to currently test our hypotheses regarding the contribution of prehospital delay and intentionality of injury to the observed mortality differences given the limitations of the variables in the databases used, namely that the Indian database does not contain prehospital mortality. However, we believe that this analysis lays the groundwork for further data collection and analysis of gender differences after trauma, especially for assault injuries.

\section{CONCLUSION}

Our study revealed that both males and females in lower-income countries have higher adjusted mortality after falls, MVCs and assaults compared with their counterparts in a higher-income country. Additionally, intentionally injured females in a lower-income country were shown to have the highest relative odds of mortality compared with any other subgroup. This demonstrates the need for the collection of prehospital and intentionality of injury data to elucidate mechanistic and sociodemographic reasons for these mortality differences. With the increased interest in building prehospital trauma capacity and trauma systems in lower-resource settings, we advocate for the need for data collection of prehospital and delay in care variables to further understand discrepancies in trauma outcomes by gender.

Contributors All authors contributed to the conception of this study and preparation of this manuscript in a significant way. MD, SDD, EM, JCP and NR were all involved in study conception and design. MD, SDS and NR were involved in data collection and analysis. MD, SDD, EM JCP and NR were all involved in data interpretation. MD prepared the manuscript and SDD, EM, JCP and NR all helped to draft, edit and modify the manuscript. All authors approve this version of the manuscript for submission.

Competing interests None declared.

Provenance and peer review Not commissioned; externally peer reviewed.

Open Access This is an Open Access article distributed in accordance with the Creative Commons Attribution Non Commercial (CC BY-NC 4.0) license, which permits others to distribute, remix, adapt, build upon this work non-commercially, and license their derivative works on different terms, provided the original work is properly cited and the use is non-commercial. See: http://creativecommons.org/ licenses/by-nc/4.0/

(c) Article author(s) (or their employer(s) unless otherwise stated in the text of the article) 2017. All rights reserved. No commercial use is permitted unless otherwise expressly granted.

\section{REFERENCES}

1. Global Burden of Disease. . The Institute for Health Metrics and evaluation. http://www.healthdata.org/gbd.

2. SuryantO, Plummer V, Boyle M. EMS Systems in Lower-Middle Income Countries: A Literature Review. Prehosp Disaster Med 2017;32:64-70.

3. Dasari M, Garbett M, Miller E, et al. Implementation of a Hospital Electronic Surgical Registry in a Lower-Middle-Income Country. World J Surg 2016;40:2840-6.

4. Zargaran E, Schuurman N, Nicol AJ, et al. The electronic Trauma Health Record: design and usability of a novel tablet-based tool for trauma care and injury surveillance in low resource settings. J Am Coll Surg 2014;218:41-50.

5. Mock C, Adzotor MB, Conklin E. Trauma Outcomes in the Rural Developing World:Comparison with an Urban Level 1 Trauma Center. J of Trauma 1993;35

6. Mock C, Arreola-Risa C, Quansah R. Strengthening Care for Injured Persons in less developed countries. A Case Study of Ghana and Mexico 2003;10:45-51.

7. Templeton J, Oakley PA, MacKenzie G, et al. A comparison of patient characteristics and survival in two trauma centres located in different countries. Injury 2000;31:493-501.

8. Haider AH, Crompton JG, Oyetunji T, et al. Females have fewer complications and lower mortality following trauma than similarly injured males: a risk adjusted analysis of adults in the National Trauma Data Bank. Surgery 2009;146:308-15.

9. Gannon CJ, Napolitano LM, Pasquale M, et al. A statewide population-based study of gender differences in trauma: validation of a prior single-institution study. J Am Coll Surg 2002;195:11-18.

10. Haider AH, Crompton JG, Chang DC, et al. Evidence of hormonal basis for improved survival among females with trauma-associated shock: an analysis of the National Trauma Data Bank. J Trauma 2010;69:537-40.

11. Laloë V. Epidemiology and mortality of burns in a general hospital of Eastern Sri Lanka. Burns 2002;28:778-81. 
12. Ahuja RB, Bhattacharya S. An analysis of 11,196 burn admissions and evaluation of conservative management techniques. Burns 2002;28:555-61.

13. Ambade VN, Godbole HV. Study of burn deaths in Nagpur, Central India. Burns 2006;32:902-8.

14. World Health Organization, Department of Reproductive Health and Research, London School of Hygiene and Tropical Medicine, South African Medical Research Council . Global and regional estimates of violence against women: prevalence and health effects of intimate partner violence and non-partner sexual violence. 2013.

15. Blackwell M, lacus S, King G. Cem: coarsened exact matching in Stata. The Stata Journal 2009;9:524-46.

16. Road Accidents in India-2015, Table 13. Ministry of Road Transport and highways. 2016 http://morth.nic.in/showfile.asp?lid=2143.

17. CIA World Factbook, https://www.cia.gov/library/publications/theworld-factbook/fields/2177.html.

18. Davis JW, Parks SN, Kaups KL, et al. Victims of domestic violence on the trauma service: unrecognized and underreported. J Trauma 2003;54:352-5.

19. Joseph B, Khalil M, Zangbar B, et al. Prevalence of Domestic Violence Among Trauma Patients. JAMA Surg 2015;150:1177-83.
20. Garcia-Moreno C. Gender inequality and fire-related deaths in India. Lancet 2009;373:1230-1.

21. Paul S. Intimate partner violence and women's help-seeking behavior: evidence from India. J of Int Econ 2016;28.

22. Shrivastava PS, Shrivastava SR. A study of spousal domestic violence in an urban slum of mumbai. Int J Prev Med 2013;4:27-32.

23. Callese TE, Richards CT, Shaw $P$, et al. Trauma system development in low- and middle-income countries: a review. J Surg Res 2015;193:300-7.

24. Kesinger MR, Puyana JC, Rubiano AM. Improving trauma care in low- and middle-income countries by implementing a standardized trauma protocol. World J Surg 2014;38:1869-74

25. Kesinger MR, Nagy LR, Sequeira DJ, et al. A standardized trauma care protocol decreased in-hospital mortality of patients with severe traumatic brain injury at a teaching hospital in a middle-income country. Injury 2014;45:1350-4.

26. Roy N, Murlidhar V, Chowdhury R, et al. Where there are no emergency medical services-prehospital care for the injured in Mumbai, India. Prehosp Disaster Med 2010;25:145-51.

27. Haider $\mathrm{AH}$, Hashmi ZG, Gupta S, et al. Benchmarking of trauma care worldwide: the potential value of an International Trauma Data Bank (ITDB). World J Surg 2014;38:1882-91. 